Chirurgia (2017) 112: 359-364

No. 4, July - August

Copyright@ Celsius

http://dx.doi.org/10.21614/chirurgia.112.4.359

\title{
Breast Cancer Surgery
}

\author{
Alexandru Blidaru \\ University of Medicine and Pharmacy "Carol Davila" , Bucharest, Romania \\ Department of Surgical Oncology, "Prof. Dr. Al.Trestioreanu" Institute of Oncology, Bucharest, Romania
}

Corresponding author:

Professor Alexandru Blidaru

University of Medicine and Pharmacy

"Carol Davila" , Bucharest, Romania

Department of Surgical Oncology,

"Prof. Dr. Al. Trestioreanu" Institute of Oncology

Bucharest, Romania

E-mail: alexandrublidaru@yahoo.com
Received: 10.07.2017

Accepted: 05.08.2017
Breast cancer treatment has been constantly improving over the past decades and the consequences of this have been an increase in survival and improvements in the quality of life of these patients. The main reasons for this are early screening and diagnosis, more accurate characterization of mammary tumours by histopathological, molecular and genomic examination in order to obtain a personalized treatment, the emergence of new molecules for systemic treatment, the improvement of radiotherapy and the progress of surgical techniques.

The "modern" history of breast cancer surgery begins, of course, with W. Halsted and the radical mastectomy type of surgery that bears his name, and which has been for nearly 50 years synonymous with the surgical treatment of breast cancer. This surgery represented the model to be followed for radical cancer treatment.

Attempts at supraradical mastectomy proposed by DahlIversen, Wangesteen, and Urban did not improve survival, and were quickly abandoned due to increased morbidity and mortality. The transition from radical mastectomy to successive forms of modified mastectomy was surprisingly slow, with every change virtually representing a revolution, even if these changes in technique were not paradigm replacements, as they were in fact faithful to the same Halstedian concept of oncological radicality. Their common desideratum was complete axillary dissection, striving to achieve this by initially preserving the pectoralis major muscle, then both major and minor pectoralis muscles. Halsted mastectomy was replaced by Patey and afterwards by Madden technique, the latter being at present the most commonly used mastectomy type (1). 
The inventiveness of the Romanian School of Surgery has manifested itself in this field as well by imagining some techniques of modified radical mastectomy.

The most well-known are: mastectomy with chimerization of the pectoralis muscles - I. Chiricuta, IOB (Bucharest Institute of Oncology) technique of transpectoral axillary dissection Al. Trestioreanu, I. Balanescu, Gh. Pitaru, the technique of repositioning of the pectoralis minor muscle after displacing it from the costal grid - D. Setlacec, and the technique of St. Maria / Grivita Hospital - P. Papahagi, M. Soare, D. Cochior, S. Constantinoiu.

At present, modified radical mastectomy remains necessary only in certain situations, mandatory in advanced stages, but not a standard, being replaced by conservative treatment (2).

Conservative treatment was accepted as an alternative to mastectomy in the 1970s, and progressively $70-80 \%$ of breast cancers have come to be treated conservatively. This was due not only to the paradigm shift in breast cancer biology, but also to the preponderance of less advanced stages at the time of diagnosis and patient desire for breast preservation, leading to the same oncological results as mastectomy, but with superior aesthetic results $(3,4)$.

The shift in the conception regarding the biology and evolution of breast cancer occurred with the theory of B. Fischer - the systemic theory and later with S. Hellman's spectral theory, so well-illustrated in Umberto Veronesi's statement: "from the maximum tolerable to the minimum effective" (18).

For a safe and visually pleasing intervention in breast cancer, several conditions have to be met: early diagnosis through screening; good quality imaging and pathological examinations; targeted systemic therapy; high-tech radiotherapy; training a surgeon or surgical team to master a wide range of surgical treatments for conservative treatment, oncoplastic surgery and reconstruction techniques (6).

Numerous randomized trials have shown that conservative treatment results in similar survival with mastectomy in cases of early stage breast cancer. It was believed that the breast cancer relapse rate is about $1 \%$ higher per year after conservative treatment compared to mastectomy, but latest studies show that both the risk of relapse and the survival rate are equivalent for the two surgical treatment modalities (7).

Oncoplastic techniques and neoadjuvant treatment have also contributed to the wider use of conservative treatment, which must be mandatorily followed by radiotherapy (8).

The extent of peritumoral breast tissue resection for conservative treatment has been long discussed. Initially a margin of $2-3 \mathrm{~cm}$ surrounding the tumour was considered to be "safe" from an oncological point of view, and the need to provide negative margins in relation to the tumour frequently led to reintervention, the rate of reintervention being as high as $50 \%$ at one point. Numerous clinical trials have demonstrated that a resection at a distance of $1-2 \mathrm{~mm}$ from the microscopic margins of the tumour is sufficient (9).

Wider excisions do not significantly reduce the risk of local relapse compared to a $2 \mathrm{~mm}$ margin, but positive margins of less than 1 $\mathrm{mm}$ double the risk of relapse of breast cancer (10).

Axillary surgery has subsequently become selective, by means of the sentinel lymph node. Unnecessary lymphadenectomy is thus avoided if the axillary lymph nodes are not invaded and in less advanced cases, in which more than $60-70 \%$ of patients do not present lymph node invasion $(11,12)$.

This method is an indisputable standard and is performed either using a radioactive tracer or a vital dye, or by combining the two techniques. Other techniques are being discussed, using fluorescent substances or magnetic nanoparticles.

Identification and biopsy of the sentinel lymph node has become standard in breast cancer and not only, and their indications have been extended not only for T1, T2, N0 stages but also for T1, T2, N1. One of the reasons for this is that clinical and imagistic assessment of the axillary lymph node condition is unreliable.

Under certain conditions, the identification of the sentinel lymph node can also be 
performed after previous interventions at the level of the breast and the axilla, after neoadjuvant treatment or in the case of multicentric tumours. The contraindications of this surgical technique have remained: advanced local stages, inflammatory forms of breast cancer, concomitant pregnancy and previous breast irradiation $(13,14)$.

Still in question is the accuracy of the sentinel lymph node technique after neoadjuvant treatment, and an even greater controversy was raised by the ACOSOG Z0011 trial, along with the hypothesis that the excision of the sentinel lymph node by itself is sufficient, even if it is invaded. The results of the trial show that there are no differences in local relapse and survival in patients with positive sentinel lymph node in whom only the sentinel lymph node was excised compared to those in whom complete axillary dissection was performed. Because of the limitations of this study, its findings were not universally accepted and this attitude has not entered common medical practice.

At present, it is considered that axillary dissection can be avoided not only in the cases of non-invasion of the sentinel lymph node, but also in cases with 1 or 2 invaded lymph nodes, where conservative treatment and postoperative radiotherapy are performed (15).

Complete axillary dissection should be avoided in the case of conservative treatment, especially since it is accompanied by morbidity, most importantly swelling of the arm and breast, and may be useless in less advanced stages of the disease, remaining necessary and obligatory in cases of extended axillary invasion, where its therapeutic role is important.

It is increasingly thought that axillary surgery is involved first and foremost in establishing the diagnosis, in staging, and as a prognostic factor, very useful in determining the appropriate course of treatment.

Another important change with implications for surgery in the treatment of breast cancer is neoadjuvant therapy. Initially, systemic preoperative treatment was only indicated in advanced locoregional stages, where surgery was not possible due to the disease extension.
This treatment attempts to reduce the mammary and axillary tumour masses with the possibility of performing surgery, mastectomy being performed in these cases for surgical wound care purposes.

The indication of systemic preoperative treatment then extended to TNM II and III stages in which modified radical mastectomy would have been technically possible as the first intention, but conservative treatment could not be performed even using oncoplastic surgery techniques.

Other potential advantages of preoperative systemic treatment in these stages would be: improving survival and assessing response to treatment. However, improvement in survival was not demonstrated if systemic treatment was performed preoperatively (neoadjuvant) compared to postoperative treatment (adjuvant) (16).

A certain advantage of neoadjuvant treatment is the ability to assess the response to treatment. The response can be clinically assessed by imagistic and histopathological means and, depending on the characteristics of the tumour and the type of treatment, can reach a complete histopathological response rate of over $50 \%$.

Survival was found to be much better in cases where a complete histopathological response was obtained.

Neoadjuvant treatment may be a challenge for the surgeon. Reducing the tumour sometimes until its disappearance makes it difficult to determine the location and extension of the breast resection. For this reason, pre-therapeutic marking of the tumours is recommended.

Patients should be informed of the possibility of obtaining a complete histopathological response seeing how serious misunderstandings and even forensic issues may arise postoperatively.

For example, if conservative treatment has been performed and no tumour is found in the resected mammary tissue, the patient assumes that the tumour site has not been excised, and if mastectomy is performed and no tumour tissue is found, doubts arise as to the accuracy of the initial cancer diagnosis. 
Preoperative information concerning these possibilities avoids their postoperative justification, at which point it can be interpreted as an excuse, or as dismissing guilt.

The reason behind surgical intervention in cases where complete histopathological response was obtained was questioned and there have been attempts to avoid it, by performing radiotherapy, but in these cases the rate of relapse was higher than in those where surgery was performed, and, furthermore, it is very difficult to establish with certainty which are the cases where complete histopathological response was obtained.

Oncoplastic surgery is the association of oncology surgery techniques with plastic surgery ones. The term "oncoplastic breast surgery" from a linguistic point of view is an oxymoron associated with a pleonasm, for those who believe that this type of surgery is performed only in breast cancer. Oncoplastic surgery can be applied in many of the localizations of neoplastic disease, an example being the treatment of cutaneous malignant tumours.

The surgeon who deals with breast cancer must possess skills of oncology surgery and plastic surgery, and in many countries is dedicated to treating only this condition, as the progress in this domain is so great that it is impossible to keep up with all new data if breast cancer surgery is not a priority in one's activity. There is a tendency to train surgeons who only operate breast cancer and thus obtain the best results both oncologically and aesthetically (17).

Oncoplastic surgical interventions can be performed by a single surgical team with both oncology and plastic surgery skills, by a mixed team consisting of an oncologist surgeon and an aesthetician surgeon, or by two successive teams: the first to operate on the cancer and the second to carry out the reconstruction, each bringing a plus to the plastic surgery and oncoplastic surgery sides of the intervention.

Some of the most important indications of oncoplastic surgery are breast cancer patients with macromastia and mammary ptosis, and those with tumours located in the internal or inferolateral quadrants (18).
Oncoplastic surgery increases the ability to perform conservative treatment, reducing the need for mastectomy, decreases the percentage of re-excision for invaded margins to allow wider mammary resection, and improves aesthetic postoperative appearance.

A classification of oncoplastic breast surgery techniques on two levels has been proposed, depending on the volume of breast tissue excised and the difficulty of the surgery.

Generally, level I techniques are used when mammary resection is less than $20 \%$ of breast volume and level II techniques are needed when the extent of breast resection needs to be larger.

Level I oncoplastic surgery includes techniques for displacing the skin, for displacing the mammary gland from the pectoral fascia, for de-epidermisation, for repositioning the nippleareola complex, or for performing glandular flaps which are mobilized to re-shape the breast (volume displacement).

Level II oncoplastic surgery is based on different replacement techniques for the removed volume with neighbouring tissues (volume replacement).

In the case of macromastia, post-treatment radiotherapy raises problems in obtaining a homogeneous dose for the entire breast. Therapeutic reductive mammoplasty allows the wide resection of cancer, the reduction of macromastia and ptosis, and, afterwards, the symmetry of the contralateral breast, the final appearance being better than before the operation. The thusly reduced breast is easier to irradiate, provided that the tumour bed and the resection margins are marked intraoperatively to accurately determine the volume for overimpression. Remodelling of the contralateral breast for symmetry can be performed during the same operative stage or, perhaps better, after radiotherapy.

Sometimes, however, it is necessary to remove the entire mammary gland due to the following reasons: multifocality, multicentricity; locally advanced breast cancer; extensive CDIS; the ratio of tumour size vs. breast size is unfavourable, even after neoadjuvant treatment; the impossibility to obtain negative 
margins; impossibility to perform postoperative radiotherapy; BRCA mutations; family history; exceptionally, the patient's wish for breast removal.

Paradoxically, in recent years, however, an increase in the percentage of mastectomies has been observed, which can be explained by the availability of mammary reconstruction and the improvement of postoperative results, especially immediate ones, the increase in the number of women who undergo genetic testing, the desire for prophylactic contralateral breast surgery to reduce the risk (which is frequently over-assessed by the patient) and anxiety, and last but not least, the more and more often use of nuclear magnetic resonance for the diagnosis of breast cancer (19).

As in other areas, in the case of breast cancer surgery we can talk about fashion and mentality.

The popularity of genetic testing for BRCA1 and BRCA2 mutations has increased significantly after the media coverage of Angeline Jolie's case, and the presence of these mutations has led to an increase in the number of unilateral or bilateral mastectomies followed by reconstruction, either immediately or at a distance of time (20).

In addition, there was an increase in contralateral mastectomy in patients with unilateral breast cancer, reaching nearly $150 \%$ in recent years, but also of bilateral prophylactic mastectomy in the absence of genetic changes (21).

In Romania more than half of the interventions performed are mastectomies, due to establishing the diagnosis in advanced stages, to the mentality of the population and of the doctors. Worldwide, the percentage of mastectomies varies between $10-15 \%$ to $80-90 \%$ (in Western European countries, in the US and Canada) of all breast cancer interventions, depending on the level of development. In other countries, like China, mastectomy is performed as elective treatment because of the small breast size.

Reconstruction of the breast after mastectomy allows for a good aesthetic result, providing a positive body image, thus reducing the psychological trauma of breast cancer patients, but there is no one-to-one answer to it (22).

Reconstruction can be performed after modified radical mastectomy, with a small cutaneous reserve in this case, or after mastectomy with skin sparing or nipple-areola sparing. Several studies confirm the oncological safety of these surgical techniques (23).

Breast reconstruction should be included in the breast cancer treatment plan when mastectomy is required. Reconstruction of the breast can be performed at the same time with mastectomy - immediate reconstruction (for less advanced stages of the disease) or at a distance from it. Alloplastic materials implants and meshes, or autologous tissue musculo-cutaneous or cutaneous-adipose flaps can be used. Several criteria are important for choosing the right type of mammary reconstruction, including: the body mass index, the appearance of the contralateral breast, the volume to be reconstructed, the patient's comorbidities, and, last but not least, the possibility of performing postoperative radiotherapy (24).

Aside from the disease stage and the oncological treatment plan, other important elements with regard to preoperative assessment and the establishment of the type of surgery are: the anatomy of the patient, the site of the tumour, the extent of the necessary breast resection and the structure of the breast.

Breast reconstruction with implants has become extremely popular due to the improvement of surgical techniques, of implant quality, and to the good aesthetic results obtained in a short operative time.

It should not be forgotten that the patient addresses the surgeon not for an aesthetic problem, but for breast cancer, and that the risk of postoperative complications is directly proportional to the extent of surgery, and these complications may delay the oncological treatment required. The minimum required for a good oncological and aesthetic result is: "keep it simple".

Breast cancer surgery in Romania is still dominated today by mastectomy, being the 
most frequent type of surgery performed. Too few patients benefit from conservative treatment and even fewer from oncoplastic surgery. Sentinel lymph node biopsy, which should be a standard, is performed in a small number of centres and in many of these the technique is still just beginning to be used. The number of breast reconstructions is still very small even after the introduction of the national reconstruction program, which partly covers the cost of the implants.

A surgeon involved in the treatment of breast cancer needs to know: various biopsy techniques; to have competence in ultrasound techniques; to know a variety of surgical techniques, including oncoplastic surgery and reconstruction; to know the indications of different types of operations and integrate them into the oncological treatment plan; to know how to perform complex surgery with good oncological and aesthetic results.

In order to achieve these goals, we consider it necessary to overspecialize in breast surgery.

\section{References}

1. Hellman S. Dogma and inquisition in medicine. Breast cancer as a case study. Cancer. 1993;71(7):2430-3.

2. Greenberg CC, Lipsitz SR, Hughes ME, Edge SB, Theriault R, Wilson $\mathrm{JL}$, et al. Institutional variation in the surgical treatment of breast cancer: a study of the NCCN. Ann Surg. 2011;254(2):339- 45

3. Veronesi U, Saccozzi R, Del Vecchio M, Banfi A, Clemente C, De Lena $\mathrm{M}$, et al. Comparing radical mastectomy with quadrantectomy, axillary dissection, and radiotherapy in patients with small cancers of the breast. N Engl J Med. 1981;305(1):6-11.

4. Veronesi U, Banfi A, Salvadori B, Luini A, Saccozzi R, Zucali R, et al. Breast conservation is the treatment of choice in small breast cancer: long-term results of a randomized trial. Eur J Cancer. 1990; 26(6):668-70.

5. Sarrazin D, Lê MG, Arriagada R, Contesso G, Fontaine F, Spielmann $M$, et al. Ten-year results of a randomized trial comparing a conservative treatment to mastectomy in early breast cancer. Radiother Oncol. 1989;14(3):177-84.

6. Litière S, Werutsky G, Fentiman IS, Rutgers E, Christiaens MR, Van Limbergen $E$, et al. Breast conserving therapy versus mastectomy for stage I-II breast cancer: 20 year follow-up of the EORTC 10801 phase 3 randomised trial. Lancet Oncol. 2012;13(4):412-9. doi: 10.1016/S1470-2045(12)70042-6. Epub 2012 Feb 27.

7. Veronesi U, Cascinelli N, Mariani L, Greco M, Saccozzi R, Luini A, et al. Twenty-year follow-up of a randomized study comparing breast-conserving surgery with radical mastectomy for early breast cancer. N Engl J Med. 2002;347(16):1227-32.

8. Fisher B, Anderson S, Bryant J, Margolese RG, Deutsch M, Fisher ER, et al. Twenty-year follow-up of a randomized trial comparing total mastectomy, lumpectomy, and lumpectomy plus irradiation for the treatment of invasive breast cancer. N Engl J Med. 2002;
$347(16): 1233-41$.

9. Bodilsen A, Bjerre K, Offersen BV, Vahl P, Amby N, Dixon JM, et al. Importance of margin width in breast-conserving treatment of early breast cancer. J Surg Oncol. 2016; 113(6):609-15. doi: 10.1002/ jso.24224. Epub 2016 Mar 16.

10. Ananthakrishnan P, Balci FL, Crowe JP. Optimizing surgical margins in breast conservation. Int J Surg Oncol. 2012;2012:585670. doi: 10.1155/2012/585670. Epub 2012 Dec 9.

11. Giuliano AE, Kirgan DM, Guenther JM, Morton DL. Lymphatic mapping and sentinel lymphadenectomy for breast cancer. Ann Surg. 1994;220(3):391-8; discussion 398-401.

12. Krag DN, Weaver DL, Alex JC, Fairbank JT. Surgical resection and radiolocalization of the sentinel lymph node in breast cancer using a gamma probe. Surg Oncol. 1993;2(6):335-9; discussion 340.

13. Patten DK, Zacharioudakis KE, Chauhan $\mathrm{H}$, Cleator SJ, Hadjiminas DJ. Sentinel lymph node biopsy after neo-adjuvant chemotherapy in patients with breast cancer: Are the current false negative rates acceptable? Breast. 2015;24(4):318-20. doi: 10.1016/j.breast. 2015.02.026. Epub 2015 Mar 21.

14. El Hage Chehade H, Headon H, El Tokhy O, Heeney J, Kasem A, Mokbel K. Is sentinel node biopsy a viable alternative to complete axillary dissection following neoadjuvant chemotherapy in women with node-positive breast cancer at diagnosis? An updated metaanalysis involving 3.398 patiens. Am J Surg. 2016;212(5):969981. doi: 10.1016/j.amjsurg.2016.07.018. Epub 2016 Aug 16.

15. Giuliano AE, Haigh PI, Brennan MB, Hansen NM, Kelley MC, Ye W, et al. Prospective observational study of sentinel lymphadenectomy without further axillary dissection in patients with sentinel nodenegative breast cancer. J Clin Oncol. 2000; 18(13):2553-9.

16. Faneyte IF, Schrama JG, Peterse JL, Remijnse PL, Rodenhuis S, van de Vijver MJ. Breast cancer response to neoadjuvant chemotherapy: predictive markers and relation with outcome. $\mathrm{Br} \mathrm{J}$ Cancer. 2003;88(3):406-12.

17. Gilligan MA, Neuner J, Sparapani R, Laud PW, Nattinger AB. Surgeon characteristics and variations in treatment for early-stage breast cancer. Arch Surg. 2007;142(1):17-22.

18. Clough KB, Kaufman GJ, Nos C, Buccimazza I, Sarfati IM. Improving breast cancer surgery: a classification and quadrant per quadrant atlas for oncoplastic surgery. Ann Surg Oncol. 2010;17(5):1375-91. doi: 10.1245/s10434-009-0792-y. Epub 2010 Feb 6.

19. Sisco M, Du H, Warner JP, Howard MA, Winchester DP, Yao K. Have we expanded the equitable delivery of postmastectomy breast reconstruction in the new millennium? Evidence from the national cancer data base. J Am Coll Surg. 2012;215(5):658-66; discussion 666. doi: 10.1016/j.jamcollsurg.2012.07.008. Epub 2012 Aug 24.

20. Evans DG, Wisely J, Clancy T, Lalloo F, Wilson M, Johnson R, et al. Longer term effects of the Angelina Jolie effect: increased riskreducing mastectomy rates in BRCA carriers and other high-risk women. Breast Cancer Res. 2015;17:143. doi: 10.1186/s13058015-0650-8.

21. Kwok AC, Goodwin IA, Ying J, Agarwal JP. National trends and complication rates after bilateral mastectomy and immediate breast reconstruction from 2005 to 2012. Am J Surg. 2015; 210(3):512-6. doi: 10.1016/j.amjsurg.2015.03.019. Epub 2015 May 14.

22. Fang SY, Shu BC, Chang YJ. The effect of breast reconstruction surgery on body image among women after mastectomy: a metaanalysis. Breast Cancer Res Treat. 2013;137(1):13-21. doi: 10.1007/ s10549-012-2349-1. Epub 2012 Dec 6.

23. Headon HL, Kasem A, Mokbel K. The oncological safety of nipplesparing mastectomy: a systematic review of the literature with a pooled analysis of 12,358 procedures. Arch Plast Surg. 2016; 43(4):328-38. doi: 10.5999/aps.2016.43.4.328. Epub 2016 Jul 20.

24. Morrow M, Li Y, Alderman AK, Jagsi R, Hamilton AS, Graff JJ, et al. Access to breast reconstruction after mastectomy and patient perspectives on reconstruction decision making. JAMA Surg. 2014;149(10):1015-21. doi: 10.1001/jamasurg.2014.548. 